\title{
Sclerosing Esophagitis with IgG4-positive Plasma Cell Infiltration
}

\author{
Shigeo Mori ${ }^{1}$, Yoshiya Tahashi ${ }^{1}$, Kazushige Uchida ${ }^{1}$, Tsukasa Ikeura ${ }^{1}$, Naoyuki Danbara ${ }^{1}$, \\ Takahiro Wakamatsu ${ }^{1}$, Takeo Kusuda ${ }^{1}$, Yu Takahashi ${ }^{1}$, Masato Yanagawa ${ }^{1}$, \\ Mitsunobu Matsushita ${ }^{1}$, Chisato Ohe ${ }^{2}$, Taku Michiura ${ }^{3}$, Kentaro Inoue $^{3}$, \\ Masanori Kon ${ }^{3}$ and Kazuichi Okazaki ${ }^{1}$
}

\begin{abstract}
:
The patient was a 76-year-old woman who had noticed slight difficulty in swallowing in the 3 years prior to this presentation. Her dysphagia progressed while she was hospitalized following cervical cancer surgery. Esophagogastroduodenoscopy and an esophagram showed circumferential erosion and a stricture of the thoracic esophagus. Esophageal resection was performed; the resected specimens showed a stricture and wall thickening. Histologically, transmural hyperplasia, which consisted of inflammatory granulation tissue with the abundant infiltration of IgG4-positive plasma cells and lymphocytes, was observed. The patient was diagnosed with probable IgG4-related disease. IgG4-related esophageal disease presenting as esophageal lesions alone is a very rare condition.
\end{abstract}

Key words: IgG4-related disease, IgG4-positive plasma cell, esophagitis, esophageal stenosis

(Intern Med 56: 3023-3026, 2017)

(DOI: 10.2169/internalmedicine.8095-16)

\section{Introduction}

IgG4-related disease (IgG4-RD) is a systemic disease characterized by fibrosing inflammation with an abundance of IgG4-positive plasma cells $(1,2)$. IgG4-RD has been found to affects the pancreas, bile duct, lacrimal glands, salivary glands, central nervous system, thyroid, lungs, gastrointestinal tract, kidney, prostate, retroperitoneum, arteries, lymph nodes, skin, and breast (2-10). Many cases of IgG4$\mathrm{RD}$ affecting the extrapancreatic organs have been reported in patients without pancreatic lesions $(11,12)$. In this case, esophageal lesions showed marked plasma cell infiltration, and immunohistochemistry revealed abundant IgG4-positive plasma cell infiltration. This pathological picture suggested sclerosing esophagitis, which was suspected to be IgG4related esophagitis.

\section{Case Report}

The patient was a 76-year-old woman who had noticed slight difficulty in swallowing in the 3 years prior to her presentation. However, prior to the worsening of her dysphagia, she had not reported experiencing heartburn, and she had not been taking any gastric acid secretion inhibitors, such as the proton pump inhibitors (PPI). Her dysphagia progressed and she began to exhibit frequent vomiting after meals and associated weight loss while she was hospitalized after surgery for cervical cancer. She had been diagnosed with diabetes at 70 years of age, underwent radical hysterectomy for cervical cancer at 75 years of age, and developed deep vein thrombosis at 75 years of age. At presentation, she had no allergies, skin disease, autoimmune disease, history of radiation exposure, and had not ingested any caustic materials; however, she was taking several medications, including lansoprazole, potassium chloride, warfarin, sen-

${ }^{1}$ Third Department of Internal Medicine, Kansai Medical University, Japan, ${ }^{2}$ Department of Pathology and Laboratory Medicine, Kansai Medical University, Japan and ${ }^{3}$ Department of Surgery, Kansai Medical University, Japan

Received: August 5, 2016; Accepted: April 17, 2017; Advance Publication by J-STAGE: October 11, 2017

Correspondence to Dr. Shigeo Mori, morishi@hirakata.kmu.ac.jp 


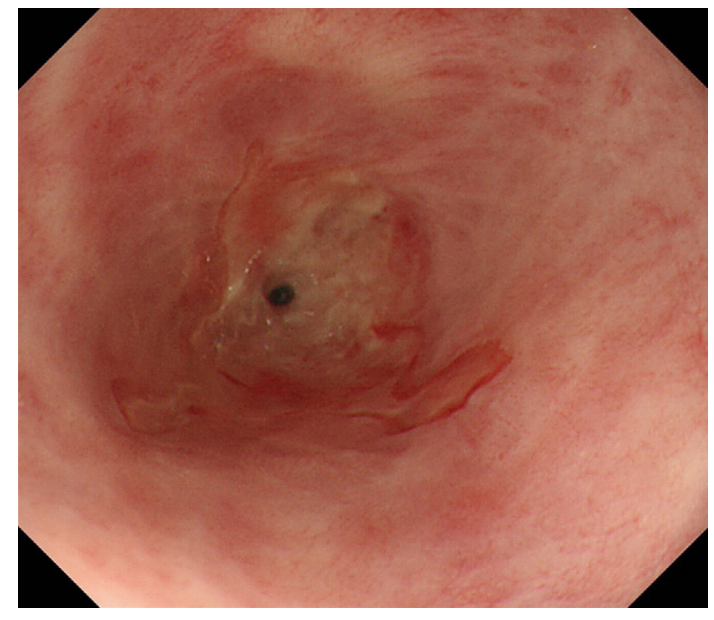

Figure 1. Esophagogastroduodenoscopy showed circumferential erosion and stricture of the thoracic esophagus. The surface of the stenotic lesion was relatively smooth, and the marginal mucosa was cloudy. Several ulcer scars were observed at the oral mucosa.

noside calcium, and levofloxacin hydrate. She began taking lansoprazole (30 mg, daily) after the worsening of her dysphagia. She had no history of tobacco smoking and only drank alcohol socially. There was no family history of cancer or autoimmune disease. On examination, she appeared uncomfortable. Her temperature was $36.6^{\circ} \mathrm{C}$, her blood pressure $134 / 81 \mathrm{mmHg}$, and her pulse was 80 beats/min. Her abdomen was soft and flat, without tenderness or distension. The other examination results were normal. Esophagogastroduodenoscopy (EGD) showed circumferential erosion and stricture of the thoracic esophagus. The surface of the stenotic lesion was relatively smooth, and the marginal mucosa was cloudy. Several ulcer scars were observed on the oral mucosa (Fig. 1). A barium esophagram showed diffuse tapered narrowing in the middle of the esophagus. Biopsy specimens from the oral surface of the stricture revealed a polynesic squamous epithelium, and inflammatory exudate and inflammatory granulation tissue with neutrophils were observed in the subepithelium. No malignant cells were identified. The laboratory results included the WBC count, $4,000 / \mu \mathrm{L}$ (reference range: $3,500-8,500 / \mu \mathrm{L}$ ); serum total protein (TP), $5.7 \mathrm{~g} / \mathrm{dL}$ (reference range: $6.5-8.2 \mathrm{~g} / \mathrm{dL}$ ); albumin (ALB), $3.4 \mathrm{~g} / \mathrm{dL}$ (reference range: 3.8-5.0 g/dL); C-reactive protein (CRP), $0.263 \mathrm{mg} / \mathrm{dL}$ (reference range: $0-0.3 \mathrm{mg} / \mathrm{dL}$ ); and squamous cell carcinoma antigen (SCC Ag), $0.6 \mathrm{ng} / \mathrm{mL}$ (reference range: $0-1.5 \mathrm{ng} / \mathrm{mL}$ ). Her postoperative serum IgG level was $640 \mathrm{mg} / \mathrm{dL}$ (reference range: $870-1,700 \mathrm{mg}$ / $\mathrm{dL}$ ) with a $\mathrm{IgG} 4$ level of $9.8 \mathrm{mg} / \mathrm{dL}$ (reference range: 4.8 $105 \mathrm{mg} / \mathrm{dL}$ ).

Computed tomography (CT) of the chest and abdomen showed circumferential esophageal wall thickening. Only the surface of the thickened lesion showed enhancement on contrast-enhanced CT, and the pancreas was normal. Her symptoms and esophageal stricture persisted despite 7 months of therapy with PPI and one endoscopic balloon dilatation. Furthermore, it was difficult to exclude the possibility of a hidden malignant tumor; thus, esophageal resection was performed. Her postoperative course was uneventful, and she was discharged on postoperative day 7 . The oral intake of food was tolerable at the time of discharge. She is currently doing well at 52 months after surgery, without evidence of disease progression to other organs. Upon gross examination, the resected specimens showed stricture with mucosal erosion and transmural wall hypertrophy measuring $50 \mathrm{~mm}$ (in length) in the middle of the thoracic esophagus. The stricture was contracted without deep ulceration (Fig. 2a). A cross-section of the site of the stricture showed wall hypertrophy from the epithelial to the submucosal layer with mucosal erosion, and the color was uniformly gray. Histologically (Fig. 2b and c), a wide area on the epithelial surface showed erosion, and tabular inflammatory lesions were subsequently formed in the subepithelium. Scattered lymphoid follicles were observed. The inflammation spread had deeply to the proper muscle layer, and severe fibrosis was present in the submucosa. Lymphocyte and plasma cell infiltration were observed in the submucosal layer and the proper muscle layer, and scattered eosinophilic infiltration was observed with up to 12 eosinophils/high power field (HPF). Neutrophilic infiltration was observed at the edge of the lesion in the proper muscle. Neither storiform fibrosis nor obliterative phlebitis was observed. Immunohistochemistry revealed IgG4-positive plasma cells at a level of $>10 /$ HPF and a ratio of $>40 \%$ IgG4+/IgG+ plasma cells (Fig. 2d). Based on comprehensive diagnostic criteria for IgG4-RD (1), the patient was diagnosed as a probable case. However, although many macrophages were observed within the lesion, they showed an atypical pattern and the accumulation of CD-163-positive spindle-shaped macrophages, which is typically observed in Type 1 autoimmune pancreatitis (13), was not identified.

\section{Discussion}

Based on the comprehensive diagnostic criteria for IgG4$\mathrm{RD}$ (1), a diagnosis of IgG4-RD is definitive in patients with organ enlargement, mass or nodular lesions, or organ dysfunction, a serum IgG4 level of $>135 \mathrm{mg} / \mathrm{dL}$, and histopathological findings of $>10 \mathrm{IgG} 4+$ cells/HPF and a IgG4 + / $\mathrm{IgG}+$ cell ratio of $>40 \%$. A diagnosis of IgG4-RD is probable in patients with organ involvement and who fulfill the histopathological criteria without increased serum IgG4 levels (1). In this patient, serum IgG4 levels were low; however, characteristic localized swelling was only found in the esophagus. Furthermore, the histopathological examination revealed $>10 \mathrm{IgG} 4+$ cells/HPF and an $\mathrm{IgG} 4+/ \mathrm{IgG}+$ cell ratio of $>40 \%$. Thus, the patient was diagnosed with probable IgG4-RD. IgG4-RD rarely develops in the gastrointestinal tract (14); however, there are reports of esophageal lesions (3-7), gastric ulcers (15) and gastric polyps (16). Lopes et al. (3) and Yang et al. (7) both reported IgG4related tumors in the esophagus. IgG4-related inflammatory 
(a)
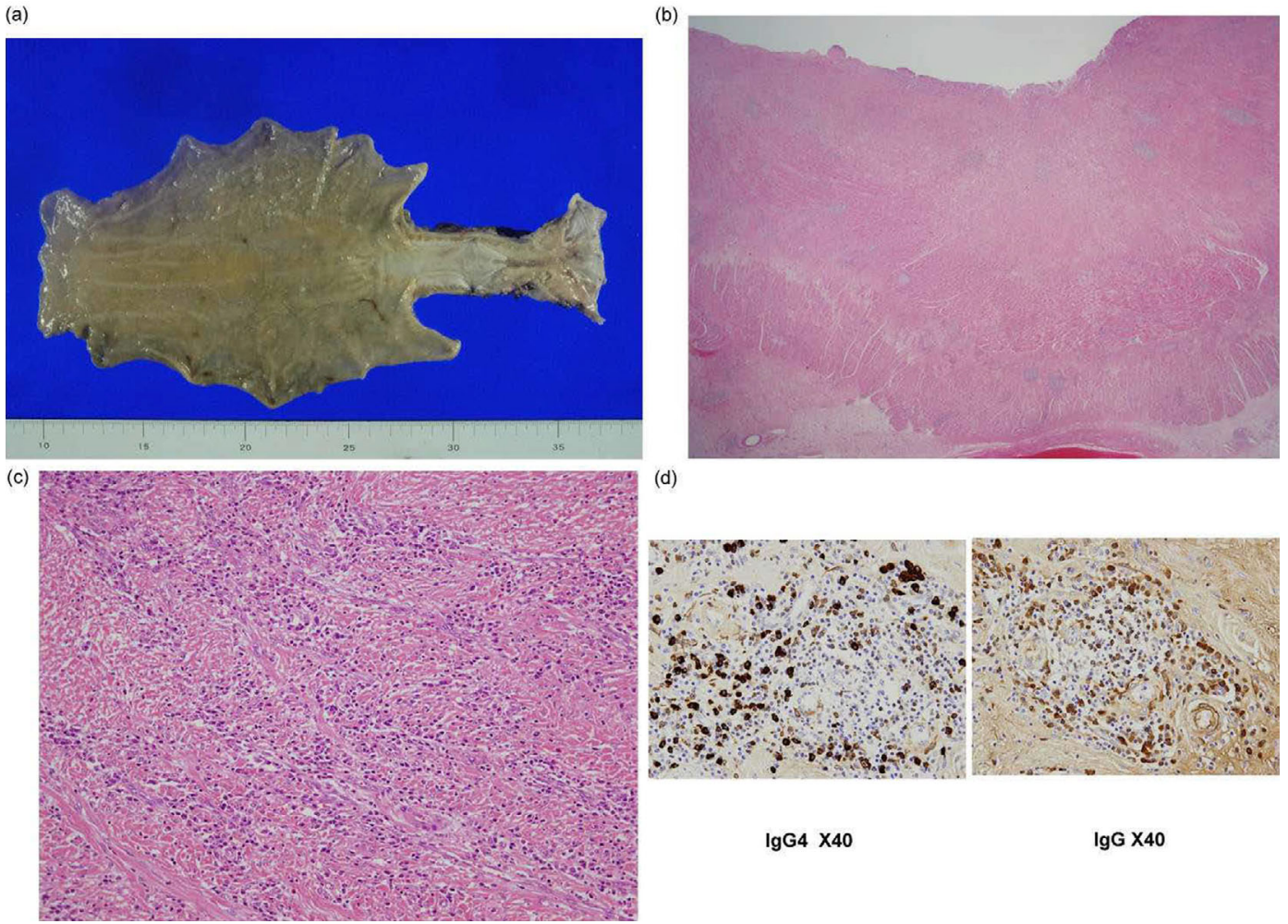

(d)

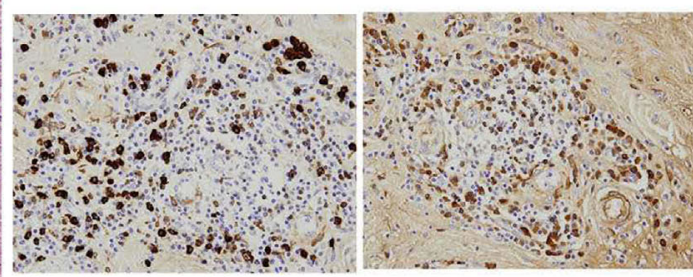

$\lg \mathrm{G} 4 \times 40$

$\lg G \times 40$

Figure 2. a: A gross examination of the resected specimens showed a stricture with mucosal erosion and transmural wall hypertrophy measuring $50 \mathrm{~mm}$ (in length) in the middle of the thoracic esophagus. The stricture was contracted without deep ulceration. $b$, c: The histopathological features. A low magnification view revealed marked inflammatory granulation tissue and fibrosis from the epithelium to the proper muscle layer. High magnification revealed abundant plasma cells and fibrosis in the submucosal layer (Hematoxylin and Eosin staining, original magnification $2 \mathrm{~b}: \times 1.25,2 \mathrm{c}$ : $\times 20$ ). d: An immunohistochemical study showed IgG4-positive plasma cells at a level of $>10 / \mathrm{high}$ powered field and the ratio of IgG4+/IgG+plasma cells was $>40 \%$.

pseudotumors (a submucosal tumor consisting of fibrosis and IgG4-positive plasma cells) are possible in such patients. Lee et al. (4), Dumas-Campagna (5), and $\mathrm{Oh}$ et al. (6) have all described IgG4-related sclerosing esophagitis. In these cases, the patients presented with stenosis, which had clinical and histopathological features that are were very similar to the present case. In three of the five cases, the serum IgG4 levels were mildly elevated. One of these cases affected the esophagus, stomach, and liver, whereas the other four were limited to the esophagus. Many diseases affecting the esophagus can cause esophagitis, including drug-induced esophagitis, escharotic stricture, gastroesophageal reflux disease (GERD), Behçet's disease (17), Crohn's disease (18), and eosinophilic esophagitis (EoE) (19). Drug-induced esophagitis has been reported after the use of antibiotics, bisphosphonates, potassium chloride $(20,21)$, non-steroidal anti-inflammatory drugs (NSAIDs), and warfarin (22). This patient had been taking antibiotics, warfarin, and potassium chloride. Many cases of drug-induced esophagitis show no histologically specific findings $(20,22)$. Thus, the abundant plasma cell infiltration, which was observed in the resected specimens in the present case, is not consistent with the classical characteristics of drug-induced esophagitis. The patient had not been experi- encing heartburn. Moreover, abundant plasma cell infiltration is not a histological characteristic feature of GERD (23). Thus, a diagnosis of GERD was excluded. Clinically and radiographically, IgG 4-RD resembles malignant tumors $(1,10)$. The symptoms and esophageal stricture persisted despite 7 months of therapy with PPI and one endoscopic balloon dilatation. Furthermore, we performed esophageal resection because the exclusion of a hidden malignant tumor was difficult. There is one report suggesting that EoE in adults is associated with $\operatorname{IgG} 4$ (24). However, our patient did not have food allergies or allergic disorders, and histologically, the number of eosinophils was $<12 / \mathrm{HPF}$. Thus, our case did not fulfill the diagnostic criteria for EoE (19). Allergen-specific IgG4 antibodies, which are induced by the administration of an allergenic extract, are known as a blocking antibodies (25). However, the association between the change in serum IgG4 antibody levels and a clinical response to a specific immunotherapy was not clear, and the association was not confirmed in a recent large-scale clinical trial (26). In the present case, we identified a stricture of the sclerosing esophagitis with IgG4positive plasma cell infiltration and diagnosed the patient with probable IgG4-RD. Interestingly, the patient only showed severe stenosis in the esophagus, and the patient's 
serum IgG4 levels were normal. Corticosteroid therapy is effective in treating $\operatorname{IgG} 4-\mathrm{RD}(2,27)$. According to the diagnostic criteria for IgG4-RD, prednisolone is recommended at an initial dose of $0.5 \mathrm{mg} / \mathrm{kg}$ per day (1). A correct diagnosis is necessary to avoid unnecessary surgery or chemotherapy. To facilitate the accurate diagnosis of this disorder and therapeutic improvement, the further collection and examination of similar cases is required.

The authors state that they have no Conflict of Interest (COI).

\section{References}

1. Umehara H, Okazaki K, Masaki Y, et al. Comprehensive diagnostic criteria for IgG4-related disease (IgG4-RD), 2011. Mod Rheumatol 22: 21-30, 2012.

2. Stone JH, Zen Y, Deshpande V. IgG4-related disease. N Engl J Med 366: 539-551, 2012.

3. Lopes J, Hochwald SN, Lancia N, Dixon LR, Ben-David K. Autoimmune esophagitis: IgG4-related tumors of the esophagus. J Gastrointest Surg 14: 1031-1034, 2010.

4. Lee H, Joo M, Song TJ, et al. IgG4-related sclerosing esophagitis: a case report. Gastrointestinal Endosc 73: 834-837, 2011.

5. Dumas-Campagna M, Bouchard S, Soucy G, Bouin M. IgG4related esophageal disease presenting as esophagitis dissecans superficialis with chronic strictures. J Clin Med Res 6: 295-298, 2014.

6. Oh JH, Lee TH, Kim HS, et al. Esophageal involvement of immunoglobulin G4-related disease: a case report and literature review. Medicine (Baltimore) 94: e2122, 2015.

7. Yang L, Jin P, Sheng JQ. Immunoglobulin G4-related disease (IgG 4-RD) affecting the esophagus, stomach, and liver. Endoscopy 47: E96-E97, 2015.

8. Kamisawa T, Okamoto A. Autoimmune pancreatitis: proposal of IgG4-related sclerosing disease. J Gastroenterol 41: 613-625, 2006.

9. Nakamura $\mathrm{H}$, Wada $\mathrm{H}$, Origuchi $\mathrm{T}$, et al. A case of IgG4-related autoimmune disease with multiple organ involvement. Scand J Rheumatol 35: 69-71, 2006.

10. Stone JH, Khosroshahi A, Deshpande V, et al. Recommendations for the nomenclature of IgG4-related disease and its individual organ system manifestations. Arthritis Rheum 64: 3061-3067, 2012.

11. Takato H, Yasui M, Ichikawa $Y$, et al. Nonspecific interstitial pneumonia with abundant IgG4-positive cells infiltration, which was thought as pulmonary involvement of IgG4-related autoimmune disease. Intern Med 47: 291-294, 2008.

12. Kim JH, Byun JH, Lee SS, Kim HJ, Lee MG. Atypical manifestations of IgG4-related sclerosing disease in the abdomen: imaging findings and pathologic correlations. AJR Am J Roentgenol 200: 102-112, 2013.

13. Fukui Y, Uchida K, Sakaguchi Y, et al. Possible involvement of Toll-like receptor 7 in the development of type 1 autoimmune pancreatitis. J Gastroenterol 50: 435-444, 2015.

14. Koizumi S, Kamisawa T, Kuruma S, et al. Immunoglobulin G4related gastrointestinal diseases, are they immunoglobulin G4related diseases? World J Gastroenterol 19: 5769-5774, 2013.

15. Fujita T, Ando T, Sakakibara M, Hosoda W, Goto H. Refractory gastric ulcer with abundant IgG4-positive plasma cell infiltration: a case report. World J Gastroenterol 16: 2183-2186, 2010.

16. Kaji R, Okabe Y, Ishida $Y$, et al. Autoimmune pancreatitis presenting with IgG4-positive multiple gastric polyps. Gastrointest Endosc 71: 420-422, 2010.

17. Hisamatsu T, Naganuma M, Matsuoka K, Kanai T. Diagnosis and management of intestinal Behçet's disease. Clin J Gastroenterol 7: 205-212, 2014.

18. Rudolph I, Goldstein F, DiMarino AJ Jr. Crohn's disease of the esophagus: three cases and a literature review. Can J Gastroenterol 15: 117-122, 2001.

19. Liacouras CA, Furuta GT, Hirano I, et al. Eosinophilic esophagitis: updated consensus recommendations for children and adults. J Allergy Clin Immunol 128: 3-20, 2011.

20. Parfitt JR, Driman DK. Pathological effects of drugs on the gastrointestinal tract: a review. Hum Pathol 38: 527-536, 2007.

21. Pemberton J. Oesophageal obstruction and ulceration caused by oral potassium therapy. Br Heart J 32: 267-268, 1970.

22. Kim SH, Jeong JB, Kim JW, et al. Clinical and endoscopic characteristics of drug-induced esophagitis. World J Gastroenterol 20: 10994-10999, 2014.

23. Yerian L, Fiocca R, Mastracci L, et al. Refinement and reproducibility of histologic criteria for the assessment of microscopic lesions in patients with gastroesophageal reflux disease: the Esohisto Project. Dig Dis Sci 56: 2656-2665, 2011.

24. Clayton F, Fang JC, Gleich GJ, et al. Eosinophilic esophagitis in adults is associated with $\mathrm{IgG} 4$ and not mediated by IgE. Gastroenterology 147: 602-609, 2014.

25. Aalberse R. The role of IgG antibodies in allergy and immunotherapy. Allergy 66: 28-30, 2011.

26. Okamoto Y, Okubo K, Yonekura S, et al. Efficacy and safety of sublingual immunotherapy for two seasons in patients with Japanese cedar pollinosis. Int Arch Allergy Immunol 166: 177-188, 2015.

27. Kamisawa T, Okamoto A. IgG4-related sclerosing disease. World J Gastroenterol 14: 3948-3955, 2008.

The Internal Medicine is an Open Access article distributed under the Creative Commons Attribution-NonCommercial-NoDerivatives 4.0 International License. To view the details of this license, please visit (https://creativecommons.org/licenses/ by-nc-nd/4.0/).

(C) 2017 The Japanese Society of Internal Medicine Intern Med 56: 3023-3026, 2017 